\title{
Should our major classifications of mental disorders be revised? ${ }^{\dagger}$
}

\author{
David Goldberg
}

\section{Summary}

Our major classification systems (DSM and ICD) face three main problems: the high rates of 'comorbidity' that are produced by our present diagnostic rules, the increasing use of 'not elsewhere classified' (NEC) by practising clinicians, and the fact that each new edition is longer and more complex than the one preceding it. A major simplification of the chapter structure used by each classification might pave the way to address these problems.

\section{Declaration of interest}

None.
David Goldberg was recently awarded the first Lifetime Achievement Award by the Royal College of Psychiatrists, and works on the Mood Disorders Group for DSM-V.

At a time when both the World Health Organization (WHO) and the American Psychiatric Association (APA) are considering modifications to our classification system of mental disorders (DSM and ICD respectively), it is timely to ask whether we have enough new knowledge to justify a major revision in the way that we group mental disorders together. Problems with both classifications include ever-increasing complexity, the very high rates of comorbidity reported in results of community surveys, ${ }^{1-3}$ and the frequent use by practising clinicians of the 'not elsewhere classified' (NEC) categories.

\section{New validating criteria for mental disorders}

The APA study group responsible for recommending modifications to the DSM system has set forth 11 aetiologically related criteria which might be satisfied before a new category is permitted. We have used these criteria to put forward a simplified model for classification, by noting larger groups of disorders that are actually quite similar when examined using these 11 criteria. ${ }^{4}$ Thus, we proposed that the 16 chapters of the DSM and the 10 chapters of the ICD can probably usefully be thought of in five large groups: neurocognitive disorders, neurodevelopmental disorders, psychoses, emotional disorders, and externalising disorders.

There is a further sixth group, disorders of bodily function such as eating, sleep and sexual disorders, where current research knowledge is not sufficient to make firm recommendations; nor have we considered personality disorders except to draw attention to the importance of certain personality disorders in determining vulnerability to the last two groups above.

A first step in reducing complexity is to use these criteria to ask what features different disorders have in common, as this may also provide a key to understanding the factors that produce

${ }^{\dagger}$ An extended version of this paper has been published in Advances in Psychiatric Treatment 2010; 16: 14-9.

D.G. was a member of the World Health Organization International Advisory Group for the Revision of ICD-10 Mental and Behavioural Disorders (Advisory Group) during 2007 to 2008, and is currently Chair of the Advisory Group's Primary Care Consultation Group. The views and opinions expressed in this manuscript are those of the author, and in no way reflect the views, policies, or other official positions of the World Health Organization, the Advisory Group, or the Primary Care Consultation Group. high rates of comorbidity. Neurocognitive disorders all have demonstrable neural substrate abnormalities and the salience of cognitive symptoms and deficits. ${ }^{5}$ The occurrence of these disorders subsequent to normal brain development sets this cluster apart from neurodevelopmental disorders where different disorders share a genetic phenotype - the disorders have an early-emerging and continuing course, and all have salient cognitive symptoms. ${ }^{6}$

The psychosis group is congruent at the level of shared psychotic psychopathology and response to antipsychotic medication, and share many biomarkers. There is modest similarity between schizophrenia and bipolar disorders relating to risk factors, neural substrates, cognition and endophenotypes, but there are key differences although these are less than those between bipolar and unipolar disorders. ${ }^{7,8}$

Externalising disorders comprise alcohol and drug dependence, antisocial personality disorder and conduct disorder, and are distinguished by the central role of disinhibitory personality. This personality type is also sometimes referred to as being low in 'constraint'. Shared biomarkers, comorbidity and course offer additional evidence for a valid cluster of externalising disorders. ${ }^{9}$

Emotional (or internalising) disorders form the largest group of common mental disorders, consisting of states with increased levels of anxiety, depression, fear and somatic symptoms. They include generalised anxiety, unipolar depression, panic disorder, phobic disorders, obsessional states, dysthymic disorders, neurasthenia, post-traumatic stress disorder and somatoform disorders. Depressive, anxious and somatoform symptoms occur together in general medical settings and share many common features, yet they are found in three different chapters of DSM-IV. ${ }^{10,11}$

In the case of emotional disorders, there are strong similarities in terms of temperamental antecedants (neuroticism or negative affect) and comorbidity, and many shared symptoms. The genes for generalised anxiety and major depressive disorder are the same; with substantial overlap for those of the fear disorders. There are also strong similarities in overall course and in response to treatment. There is incomplete evidence for somatoform disorders and for neurasthenia, but this is not because there is contrary evidence; it is because the necessary research appears not to have been done.

\section{Plato v. Aristotle}

Those who believe in a Platonic ideal of categorical diagnoses ${ }^{12}$ may take the view that with each revision of the classification we move towards a system which is mutually exclusive and jointly 
exhaustive. However, most mental disorders are continuously distributed in the general population, as are hypertension and anaemia. In the case of hypertension, the threshold which defines the disorder has moved appreciably in the past 50 years, to take account of improvements in both investigation and treatment. Similar arguments apply to the emotional disorders.

Those who prefer an Aristotelian approach to diagnosis ${ }^{12}$ remind themselves that not only are the various disorders separated from normality by arbitrary thresholds, but the various categories merge into one another, so that 'points of rarity' are rare indeed. In this view, the most that we can hope of our current diagnostic system is that it should take account of advances in research knowledge that have occurred since the classification was last revised.

The changes advocated above have taken such knowledge into account, but freely acknowledge that our groupings are provisional, and must be ready for revision as more information becomes available. Critics of the proposed changes may take the view that they are a step backwards towards a time when the great distinction was between neuroses and the psychoses, and that the grounds for such a distinction were never satisfactorily stated. The major change in the present proposals is that our system should take account of the importance of personality variables in determining emotional and externalising disorders, and there is clearly room for much expansion of this approach in the field of common mental disorders. It has been necessary for psychiatrists to loosen their grip on categorical types of personality disorders in favour of less static dimensional approaches, and for clinical psychologists to abandon their mutual recriminations about how many dimensions of personality are important in describing normal variations.

There are also problems in depriving child psychiatry of a fully comprehensive diagnostic system, by assigning conduct disorder to externalising disorders and anxiety disorders (now in the emotional disorders). Childhood disorders may indeed manifest themselves differently at different ages: for example, pre-pubertal anxiety may be followed by an episode of adolescent depression, as the adolescent confronts major problems in peer popularity, educational achievement or sexual choice. Neither is there always a linear relationship between childhood problems and adult disorder; conduct problems at ages 7-9 years may be associated with increased risk for antisocial personality disorder and crime in early adulthood (ages 21-25 years), but also with adverse sexual and partner relationships (including domestic violence), early parenthood, and increased risks of substance misuse, mood and anxiety disorders, and suicidal acts. ${ }^{13}$ In the Dunedin study, for example, conduct problems at ages 11-15 were associated with increased risk for all psychiatric disorders at age 26, including internalising problems, schizophreniform disorders and mania, in addition to broadly externalising phenomena such as substance abuse. ${ }^{14}$

Where mental disorders in general medical practice are concerned, one solution to the NEC problem would be to allow clinicians to describe the patient's main presenting problems, allowing such diagnoses as 'anxious depression' or 'depression with somatic symptoms' rather than just 'depression'. Failure to note that a patient with depression is morbidly anxious may result in not prescribing the optimal psychotropic, not receiving the optimal form of cognitive-behavioural therapy or, most important of all, not receiving reassurance for symptoms that are troubling the patient, but which are ignored because they are not part of the category being diagnosed. Failure to note that a patient with depression has somatic symptoms may cause clinicians to neglect to give the patient any explanation of the symptoms which are sometimes alarming them most of all. The present DSM classification puts major depression, anxiety disorders and somatoform disorders in three different chapters, while the ICD has them in two, necessitating multiple 'comorbid' diagnoses.

In conclusion, to what extent do these changes meet the three problems mentioned at outset? They undoubtedly provide at least a partial solution to the increasing complexity of both classifications, as they provide a major simplification in the overall structure, and to this extent also a partial solution to the comorbidity problem. To take matters further with this problem, it would be necessary to adopt the solution described above when multiple categories are mentioned within a particular group of disorders. The NEC problem has been partly solved already by the ICD-10 classification, which allows three grades of severity of depression, as well as anxious depression, where both are subthreshold but meet criteria between them, as well as the combination of depression with the 'somatic syndrome'. This does not apply to the DSM-IV classification; however, various plans for rating dimensions such as anxiety and suicidality, in addition to main category diagnoses, have been discussed. A full dimensional scheme, where every diagnosis allows 'subthreshold' degrees of severity, may well turn out to be a bridge too far, as it would in effect greatly increase the overall prevalence of mental disorders in the population.

David Goldberg, FRCPsych, Health Service and Population Research, Institute of Psychiatry, King's College London, De Crespigny Park, London SE5 8AF, UK. Email: David.Goldberg@iop.kcl.ac.uk

First received 17 Nov 2009, accepted 21 Dec 2009

\section{References}

1 Kessler R, Chiu WT, Demler O, Walters EF. Prevalence, severity and comorbidity of 12-month DSM-IV disorders in the National Comorbidity Survey Replication. Arch Gen Psychiatry 2005; 62: 617-27.

2 Vollebergh WAM, ledema J, Bijl RV, de Graaf R, Smit F, Ormel J. The structure and stability of common mental disorders: the NEMESIS study. Arch Gen Psychiatry 2001; 58: 597-603.

3 Andrews G, Anderson TM, Slade T, Sunderland M. Classification of anxiety and depressive disorders: Problems and solutions. Depress Anxiety 2008; 25: $274-81$.

4 Andrews G, Goldberg DP, Krueger RF, Carpenter WT, Hyman SE, Sachdev, P, et al. Exploring the feasibility of a meta-structure for DSM-V and ICD-11: could it improve utility and validity? Psychol Med 2009; 39: 1993-2000.

5 Sachdev $P$, Andrews G, Hobbs MJ, Sunderland M, Anderson TM. Neurocognitive disorders: cluster 1 of the proposed meta-structure for DSM-V and ICD-11. Psychol Med 2009; 39: 2001-12.

6 Andrews G, Pine DS, Hobbs MJ, Anderson TM, Sunderland M. Neurodevelopmental disorders: cluster 2 of the proposed meta-structure for DSM-V and ICD-11. Psychol Med 2009; 39: 2013-23.

7 Carpenter WT, Bustillo JR, Thaker GK, van Os J, Krueger RF, Green MJ. The psychoses: cluster 3 of the proposed meta-structure for DSM-V and ICD-11. Psychol Med 2009; 39: 2025-42.

8 Goldberg DP, Andrews G, Hobbs MJ. Where should bipolar disorder appear in the meta-structure? Psychol Med 2009; 39: 2071-81.

9 Krueger RF, South SC. Externalizing disorders: cluster 5 of the proposed meta-structure for DSM-V and ICD-11. Psychol Med 2009; 39: 2061-70.

10 Goldberg DP, Krueger RF, Andrews G, Hobbs M. Emotional disorders: cluster 4 of the proposed meta-structure for DSM-V and ICD-11. Psychol Med 2009; 39: 2043-59.

11 Löwe B, Spitzer RL, Williams JBW, Mussell M, Schellberg D, Kroenke K. Depression, anxiety and somatization in primary care: syndrome overlap and functional impairment. Gen Hosp Psychiatry 2008; 30: 191-9.

12 Goldberg DP. Plato versus Aristotle: categorical and dimensional models for common mental disorders. Compr Psychiatry 2000; 41(suppl 1): 8-13.

13 Fergusson DM, Horwood LJ, Ridder EM. Show me a child at seven: the consequences of conduct problems in childhood for psychosocial functioning in adulthood. J Child Psychol Psychiatry 2005; 46: 837-49.

14 Kim-Cohen J, Caspi A, Moffitt TE, Harrington H, Milne BJ, Poulton R. Prior juvenile diagnoses in adults with mental disorders: developmental followback of a prospective-longitudinal cohort. Arch Gen Psychiatry 2003; 60: 709-717. 